\title{
Correction to: Post-transcriptional Regulation of Cytokine Signaling During Inflammatory Responses
}

Irina Vlasova-St. Louis and Paul R. Bohjanen

\section{Correction to:}

Chapter 3 in: K. M. J. Menon, A. C. Goldstrohm (eds.), Post-transcriptional Mechanisms in Endocrine Regulation, https://doi.org/10.1007/978-3-319-25124-0_3

The original online version of this chapter was inadvertently published with part of last name of the author "Irina Vlasova-St. Louis" included along with the first name. The correct first and last name of this author is given below:

First name: Irina

Last name: Vlasova-St. Louis

The updated online version of this chapter can be found at https://doi.org/10.1007/978-3-319-25124-0_3 